\title{
LA MODIFICACION DEL BIOTOPO PERIHABITACIONAL EN LA PROFILAXIS DE LA ENFERMEDAD DE CHAGAS
}

\author{
Miguel Eduardo Jörg
}

\begin{abstract}
La modificación del biotopo perihabitacional consiste en crear alrededor de la vivienda humana, rural, selvática o suburbuna, un espacio perimetral limpio, totalmente libre de malezas y chaparral, despojado de nidos, madrigueras o refugios de animales silvestres y de habitáculos de animales domésticos; de suficiente magnitud para evitar que tras desinsectación, queden focos peridomiciliarios de proliferacción de triatomineos, vectores del Trypanosoma cruzi, focos inmediatos y habituales de reinfestación domiciliaria por estos redúvidos hematofagos. Los trabajos de rociado entomicida y aún el mejoramiento de la vivienda se reducen extremadamente si no son complementadas con la modificación del biotopo perihabitacional. Se expone una experiencia piloto sobre un barrio de la ciudad Villa Carlos Paz, provincia Córdoba, Argentina, que en 2 años de aplicación permitió verificar la desaparición de vinchucas en un area de baja desindad de vectores y hospedadores.
\end{abstract}

Palabras-claves: Enfermedad de Chagas. Triatoma infestans. Modificación del biotopo perihabitacional. Control de vectores.

En 1938, Salvador Mazza, en comunicaciones al VI Congreso Nacional de Medicina (Córdoba, Argentina), partiendo de su experiencia ${ }^{6}$ y de lo aportado por los estudiosos brasileños Evandro Chagas y Emmanuel Dias a la 9a Reunión de la Sociedad Argentina de Patologia Regional (Mendoza, 1935) complementado por lo expuesto por César F. Pinto en su visita a la Misión de Estudios de Patología Regional Argentina (Jujuy, 1936), expuso que las medidas más plausibles y racionales para la prevención y aun la erradicación de la enfermedad de Chagas podrian ser enunciadas en los siguientes principios generales de alcance sanitario:

1) Es necesario proceder a estudios y medidas de acción general para lograr la modificación fundamental de la vivienda rural y ciudadana infestada por vinchucas o susceptible de serlo. Esto requiere la eliminación del rancho con todas sus implicancias de deformación social y familiar, concomitante con planes que faciliten la construcción de viviendas higiénicas, adaptadas tanto a las posibilidades materiales y economicas, como al uso, necesidades, clima y particularidades de cada región y cada comunidad. 2) Es menester poner en marcha un programa y una acción de Educación Sanitaria, en todos los niveles culturales, desde el popular al universitario, pero inicial - y fundamentalmente, por el escolar primario y el trabajo social-sanitario directo en las areas de endemismo dominante. El término final es llevar el

Endereço para correspondência: Dr. Miguel Eduardo Jörg. Centro Medico de Mar del Plata. San Luis 1978. 7600 Mar del Plata, Argentina.

Recebido para publicação em 15/3/89. conocimiento de la enfermedad, sus riesgos y las posibilidades de profilaxis a la mayor masa de población posible.

En 1940, Mazza y Jörg (dados não publicados) partiendo de ensayos realizados con los primeros insecticidas sintéticos de al to poder que les habían sido confiados para prueba, ampliaron las proposiciones anteriores, agregándole lo siguiente: 3) Requiérese cortar la cadena morbígena, actuando sobre el punto más accesible: impedir el desarrollo domiciliario de la vinhuca y destruirla en todos sus períodos de desarrollo en la habitación humana y su entorno, con insecticidas los más específicos posibles, preferentemente de acción prolongada, de costos accesibles para grandes campañas y de mínimo riesgo en cuanto a toxicidad para el hombre y los animales domésticos.

A esta premisa y fundándose en un ensayo regional, Jörg 45 amplió los conceptos anteriores con lo siguiente: 4) Es indispensable completar las medidas enunciadas, con la modificación del biotopo perihabitacional, para alejar a los hospedadores y transmisores silvestres y domésticos de la vivienda humana; además para evitar que durante los rociados insecticidas de las casas, las vinchucas encuentren refugio en el entorno y, en suma, para crear, en el más amplio alcance y sentido, condiciones ecológicas generales adversas para la perpetuación de la cadena morbigena de la tripanosomiasis cruzi.

\section{Definiciones y planteo operacional}

La modificación del biotopo perihabitacional consiste en lograr el reemplazo del ámbito selvático, o montaraz, o simplemente basurero y desordenado que suele rodear la habitación humana primitiva de nues- 
tras areas rurales; ámbito perihabitacional plagado de nichos ecológicos que permiten la perpetuación de aves y mamiferos que, a su vez, son centros de cria y desarrollo de triatóminos, silvestres y domésticos; focos a partir de los cuales se produce la infestación y la reinfestación tras expulgos de las moradas vecinas.

Estos nichos ecológicos, muchas veces verdaderas microciudades artropódicas (alojan ácaros, arañas, escorpiones, garrapatas y la más variada gama de hexápodos) son: ya el gallinero o el palomar rústico, ya el primitivo corral de ganado caprino u ovino, ya nidos de pájaros, pajareras o jaulones - en especial de psitácidos o dendrocoláptidos -, ya cuevas o refugios de mamíferos, sean roedores, murciélagos o zarigüeyas. A ellos se asocian como hospedadores parasitarios y alimentadores de las vinchucas, perros, gatos y aún animales silvestres domesticados como coaties, cuyes, etc.

La modificación del biotopo perihabitacional es pues una propuesta de saneamiento antitriatomínico. Lo que se logra creando alrededor de la casa cualquiera sea su tipo - un área "limpia", libre de vinchucas y libre de todo lo que pueda servirles de refugio, tanto al mismo insecto como a los animales que le sirven de fuente alimentaria. Exige esta area limpia que a la casa no tengan acceso ni perros, ni gatos, ni aves, ni animales silvestres. Requiere que se limpie el espacio perihabitacional de malezas, cuevas de roedores, nidos de pájaros y de cuanta basura o desecho, $o$ acumulación de leña y materiales en desuso exista y que puede servir de refugio a insectos o animales asociados.

La eficacia de los expulgos, de la lucha antitriatomínica por rociado o fumigación con insecticidas, se reduce extraordinariamente si no es complementada por el saneamiento perihabitacional, ya que sin éste, la reinfestación por vinchucas se facilita en grado sumo. Un hecho biológico justifica esta afirmación: la cría y desarrollo de la vinchuca casera de la Argentina, el Triatoma infestans, es más fácil y más rápida en los nichos ecológicos animales que en la habitación humana, porque en ellas trascurre mejor el desarrollo de las ninfas primarias de los triatóminos; el punto más débil de la etología de ese insecto. El parasitismo hematofágico de las vinchucas en la habitación humana es realizada prevalentemente por los insectos adultos $\mathrm{y}$ las ninfas terminales - ambos ágiles $\mathrm{y}$ resistentes - dotados de gran movilidad y capacidad de agresión. Las ninfas I y II son frágiles y de menor movilidad; su desarrollo es mucho más fácil en nichos ecológicos animales, sobre todo si hay crias y pichones, sobre las cuales se alimentan sin dificultades, sin riesgos de movimientos de defensa de los atacados. Inclusive las ninfas menores pueden ocultarse con facilidad bajo el plumaje denso de ciertas aves; lo hemos verificado en gallinas y palomas; sobre todo en gallinas cluecas cuando están empollando o cubriendo ya a polluelos. En las cuevas de roedores tales como los Hesperomys, Hypsimys, Eligmodontia, del subdistrito zoológico jujeño-tucamano, hemos encontrado una desindad de ninfas de triatominos muy superior a la que puede verificarse en una habitación humana salvo ranchos muy primitivos y llenos de animales a la par del hombre. Los nichos ecológicos perihabitacionales son pues verdaderas "incubadoras" de vinchucas, desde las cuales infesta y reinfesta la morada humana. En nuestra experiencia, el Triatoma infestans se desarrolla sólo excepcionalmente en nichos ecológicos selváticos o montaraces; su climax ecológico es la habitación humana y sus dependencias; aunque la capacidad de dispersión de la vinchuca adulta es muy grande y se la puede hallar lejos de tal sitial.

El problema fundamental de la modificación del biotopo perihabitacional reside en determinar qué amplitud o extensión debe tener el area "limpia", excento de incubatorios de vinhucas. No es posible afirmar, a priori, medidas uniformes para todas las regiones, disposiciones y tipos de congregaciones habitacionales. Corresponde - de acuerdo al consenso de los estudiosos citados - realizar estudios in situ para establecer;

(1) Qué animales, qué vegetales y sus asociaciones bióticas crean nichos ecológicos de triatomineos. Esto se extiende a la misma casa o habitación, pues se crían vinchucas bajo los tejados, en el espacio del entretecho, v.d. entre el entablonado y su revestimiento externo con filetro embreado y las tejas, a veces con pájaros, murciélagos y aún roedores pequeños; igualmente en las cajas de las cortinas de arrollar se han encontrado criaderos de vinhucas con nidos de pájaros e murciélagos.

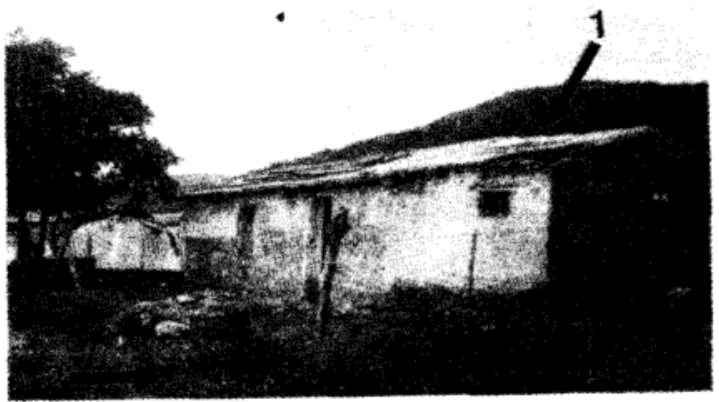

Figura 1. La paradoja triatominica! En el interior de esta construcción, vivienda de obreros de una empresa agropecuária, no se encontraron vinchucas; sin embargo las habia en el entretecho abierto (flecha 1) refugio de murciélagos, ejemplares de Triatoma platensis. Se entraron tambien ejemplares en una madriguera de zarigüeya y ratones en el matorral vecino a la casa (flecha 2).

(Observación Villa Carlos Paz, 1966) 
Jörg ME. La modificación del biotopo perihabitacional en la profilaxis de la enfermedad de Chagas. Revista da Sociedade Brasileira de Medicina Tropical 22: 91-95, Abr-Jun, 1989.

(2) Qué tipo de transformación es la más adecuada y más adaptable a los medios regionales para ser realizada y mantenida. Suele crear dificultades a los moradores, la necesidad de alejar un gallinero o un corral de cabras; ante la imposibilidad del distanciamiento debe pensarse en una racionalización "limpia" de tales instalaciones.

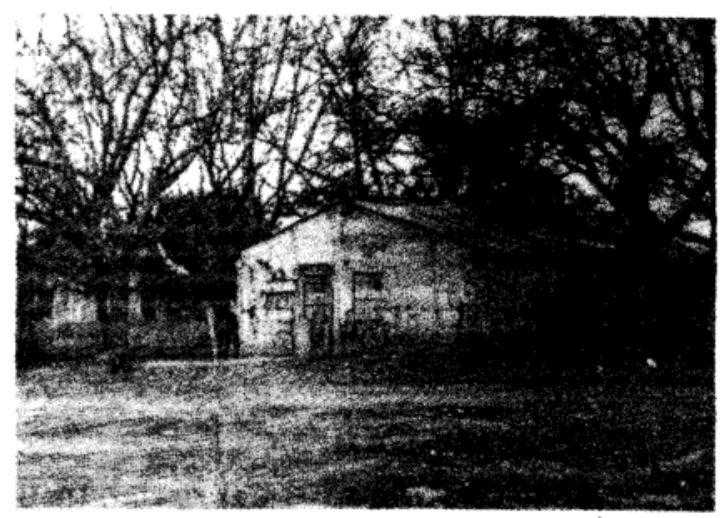

Figura 2. Vivienda de obreros de una empresa constructora vial. La limpieza parcial del entorno a la vivienda logró que la invasión de vinchucas a la casilla se hiciera excepcional, pese a la rusticidad de la misma. (Observación Villa Carlos Paz, en las lindes del monte serrano 1966)

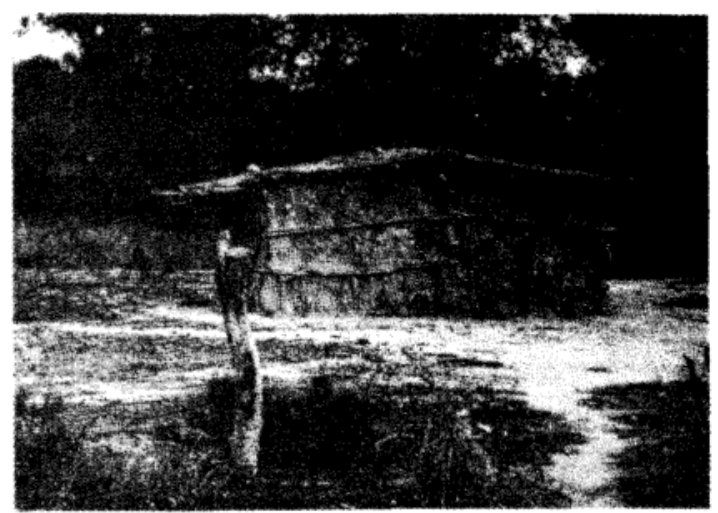

Figura 3. Rancho tipico de alto primitivismo, paredes de barro, techo de ramas, y pasto, vivienda de obreros leñateros. La rudimentaria limpieza en torno al mismo logró reducir al minimo la reinfestación por T.infestans tras una precaria desinsectación. (Observación Villa Carlos Paz, 1966, en area montaraz)

(3) Qué extensión y amplitud, disposición y caracter, es conveniente para el area limpia teniendo en cuenta el entorno montaraz o selvático.

Una serie de pruebas y ensayos sobre el campo nos demostraron que un cinturón de 20 metros, rigurosamente libre de criaderos de vinchucas en torno a una casa o un rancho es la dimensión mínima del area de saneamiento. Esta distancia puede ser atravesada por las formas adultas voladoras del Triatoma infestans, pero no es cruzada por las formas ninfales. Produce asi una disminución significativa de la magnitud de infestación o de la reinfestación por triatomineos tras expulgos.

\section{MATERIAL E MÉTODOS}

Por nuestra iniciativa y la cooperación asidua de los profesores Felix Ramacciotti y Enso Criscuolo de la Universidad de Córdoba, se realizó entre los años 1963 y 1969 una tentativa de reducción del índice triatomínico en un area marginal urbana de la localidad Villa Carlos Paz, provincia Córdoba, Argentina. Se tomó un area de 600 metros por 1500 metros, ocupado prevalentemente por casas de material, ladrillos y techos de tejas, 14 unidades de las cuales sólo 2 tenían cubreventanas de tela antimosca; además 6 casas de construcción más primitiva, con paredes no revocadas o techos rudimentarios. Se eligió tal area pues se habia verificado que en la mayoria de las casas se atrapaba un promedio de 4 vinhucas de desarrollo completo, voladoras, ya atraidas por faroles en los portales de las casas o tratando penetrar a las mismas, cada dia durante el verano. 12 perros y 8 gatos domésticos formaban la problación de mamíferos domésticos. Habia 6 gallineros menores, muy primitivos, en los fondos de los lotes.

Mediante la cooperación de los miembros de una Sociedad de Fomento Vecinal se inició primero una campaña de apalabramiento y concientización de los vecinos, casa por casa, haciéndoles conocer el riesgo de la presencia de vinchucas. No se contó con apoyo oficial, pues siendo la localidad una villa de turismo se temió que la campaña pudiera actuar en desfavor de la concurrencia de visitantes, creando temor. Por ello en la campaña se habló siempre de "mejoramiento urbano" reservando la realidad a las entrevistas verbales con los moradores del area. Se obtuvo cooperación activa de $85 \%$ de los vecinos.

\section{RESULTADOS}

Se logró la eliminación de todos los gallineros, menos uno de una persona que vivía de la venta de aves y huevos. Se le ayudó a construir un gallinero antivinchuva y se consiguió que los dueños la pintaran periódicamente con lechada de cal y en oportunidades con $\mathrm{HCH}$.

Se consiguió que todos los vecinos con casas tipo chalet, trasformaran sus lotes en parques, eliminando todo malezal, quitando arbustos silvestres y plantas rastreras, plantando árboles de jardín en especial coniferas, paraisos y eucaliptos. Se obtuvo la reducción del número de perros a 4 y se eliminaron los 
gatos menos uno. Se trampearon roedores en forma continua y se cazaron ${ }^{6}$ zarigüeyas Didelphis albiventris que de noche acudian a revolver tarros de residuos o invadían los gallineros.

Esta fase fue terminada en el primer verano tras su iniciación. Al verano seguiente, extremando la limpieza a los baldios, quemando el malezal, eliminando residuos, destruyendo cuevas de roedores y asegurando que una vez por mes cada terreno fuera desmalezado, se logró que el número de vinchucas atrapadas por casa que en el primer mes era menor a 1 espécimen por casa/dia, llegara a ser excepcional al final de la estación estival.

Vivimos 6 años en el lugar; despues del $2^{\circ}$ año de la campana, no se hallaron más vinchucas, pese a que 200 metros por fuera del area de trabajo existía una ranchada con establos, caballos, gallinas sueltas, dos ovejas y todo géneros de animales domésticos, peros, gatos y conejos. Vivían allí gente muy rústica que se negó a toda cooperación.

\section{DISCUSSÃO}

La experiencia referida pone en evidencia quepor cierto en región no estrictamente endémica para la tripanosomiasis cruzi - fue posible eliminar en un barrio la presencia de vinchucas. Cabe agregar que todas las vinchucas atrapadas no presentaban infestación por el Trypanosoma cruzi. Asimismo todos los mamíferos tanto silvestres como domésticos cuya sangre fuera examinada al microscopio tras concentración por el método Strout-Yaeger, resultaron libres de infección por Trypanosoma cruzi. El hecho no extranó, pues Villa Carlos Paz está fuera del area endémica para la tripanosomiasis en Córdoba; el Dr. José R Ibánez, especializado en el estudio de la enfermedad de Chagas nos hizo presente, en reiteradas comunicaciones personales, que durante 20 años de residencia local no había observado casos agudos de la dolencia originados en la región. Vale decir que se trabajó en una región con triatomismo pero sin tripanosomiasis.

Un estudio de C. A. Soler ${ }^{7}$ ilustra con detalles y cifras estadisticas la curva de reinfestación de habitaciones por $T$. infestans tras expulgos y analiza los diversos mecanismos que en ella intervienen. Un estudio ulterior del mismo investigador (dados não publicados) pasa en revisión prolija la frecuencia y estructura de los focos peridomésticos como fuente de reinfestación triatomínica de la vivienda humana, exponiendo cifras significativas de infestación del Triatoma infestans por el Trypanosoma cruzi en gallineros y corrales de cabras, demonstrando inequívocamente que existe intercambio de vinchucas entre dichos focos y las habitaciones de los hombres. Ambas publicaciones y sus conclusiones abonan firmemente la necesidad de modificación del biotopo perihabi- tacional en una campaña integral de expulgo vectorial para combatir la enfermedad de Chagas.

Destacamos que la modificación del biotopo perihabitacional es sólo una medida complementaria de todo lo demás que incumbre a la prevención de la tripanosomiasis cruzi: mejora de la habitación humana, desinsectación y creación de una conciencia de limpieza, orden y disciplina sanitarias en la vivienda. Pero tambien recalcamos que sin la modificación del biotopo aludido, sin el alejamiento de los nichos ecológicos pro-triatominicos vecinos a la casa; impidiendo a la par el acceso de mamiferos a la misma y eliminando los refugios de vectores en la misma vivienda, no se logrará la desinsectación integral y duradera. Añadimos que la Educación Sanitaria es una necesidad imperiosa simultanea, para el desarrollo de una conciencia preventiva popular, factor primordial para mantener y ampliar las otras medidas. Ya que de nada valen las viviendas higiénicas - en reemplazo del rancho - y la desinsectación jamás será fructífera si los habitantes no conservan sus moradas en condiciones óptimas de limpieza, sin permitir ni provocar su deterioro, sin arrancar - con el pretexto que reducen la ventilación-los cubreventanas y sobrepuertas de tela antiinsectos, reparando las roturas y grietas, denunciando prestamente la reinfestación cuando ella se produce y no puede ser combatida por los propios moradores.

Tenemos la seguridad que la medida profiláctica complementaria aquí expuesta es conocida ampliamente por los estudiosos brasileños que se ocupan del tema, por lo cual estimamos que esta modesta contribución vendrá en apoyo de las importantes campañas que se han realizado en Brasil, especialmente por iniciativa y conducción de João Carlos Pinto Dias 123.

La importancia de la modificación del biotopo perihabitacional ha sido reconocida por los expertos en el control de campaña de los vectores de la enfermedad de Chagas, como lo demuestra su inclusión en el temario de una reciente reunión de un grupo de trabajo del Programa Especial de UNDP/Banco Mundial/Organización Mundial de la Salud para la Investigación y Adiestramiento en Enfermedades Tropicales (sigla TDR) ${ }^{8}$. 


\section{SUMMARY}

In zones endemic for the American trypanosomiasis the modification of the biotopes surrounding human, rural, sylvatic or suburban housing, involves the arrangement of a clean perimetral area completely free of shrubs and chaparral, devoid of dens of wild animals and dwellings of domestic animals, to hinder the persistence of peridomestic foci where the proliferation of Triatomine bugs encourage the reinfestation of the human lodgings. The success of insecticide spraying campaigns, even with simultaneous amelioration of housing, are enhanced by the modification of the biotopes. A pilot field experience is presented: a 2 year active campaign in a suburban area of Villa Carlos Paz, province Cordoba, Argentina, confirming the methods to eliminate reinfestation of houses from peridomestic sites, in an area of low vector density.

Key-words: Chagas'Disease. Triatoma infestans. Peridomestic environmental management. Vector control.

\section{REFERÊNCIAS BIBLIOGRÁFICAS}

1. Dias JCP, Dias RB. Las viviendas y la lucha contra los vectores de la enfermedad de Chagas en el hombre, en el
Estado Minas Gerais, Brasil. Boletín de la Oficina Sanitaria Panamericana 93:453-465, 1982.

2. Dias JCP. Historia natural de la enfermedad de Chagas. In: Cançado JR, Chuster Med: Cardiopatia chagasica. Ed. Fundação Carlos Chagas, Belo Horizonte p. 99-113, 1985.

3. Dias JCP. Reseña histórica de los conocimientos sobre la enfermedad de Chagas y reflexiones sobre algunos aspectos políticos y socioeconómicos de la endemia en el contexto latinoamericano. (Relato al Simposio Latinoamericano sobre Enfermedad de Chagas, Córdoba 1987). Revista de la Federación Argentina de Cardiologia 17:128-135, 1988.

4. Jörg ME. Ecologia sanitaria. Su component socioeconómico y la erradicación de la tripanosomiasis cruzi. Archivos Argentinos de Medicina 3:56-78, 1958.

5. Jörg ME: Anotaciones criticas sobre enfermedades parasitarias en Argentina; alcance de la educacion sanitaria e la prevención. Archivos Argentinos de Medicina 6:15-17, 1961.

6. Mazza S. Enfermedad de Chagas. Actualidad en nuestra experiencia y perspectivas. Actas del 6. Congresso Nacional de Medicina, Córdoba, 3:160, 1939.

7. Soler CA. Reinfestación con T. cruzi de las viviendas desinsectadas por HCH. La Rioja 1965. Revista de la Universidad Nacional de Córdoba 10:1293-1316, 1969.

8. WHO. Organización Mundial de la Salud: Report of a meeting.on research needs in the field of Chagas'disease vector control. Panamá, September 1987. Boletín TDR\&CHA/PAN. 87.31988. 